\title{
Simplified Fuzzy Model based Predictive Control for a nonlinear system
}

\author{
Juan M. Escaño, Carlos Bordons \\ Departamento de Ingeniería de Sistemas y Automática. Universidad de Sevilla \\ jescano/bordons@us.es \\ Samira Roshany-Yamchi, Kritchai Witheephanich, Jean Michel Rubillon \\ Nimbus Centre. Cork Institute of Technology \\ samira.roshany/kritchai.witheephanich/jeanmichel.rubillon@cit.ie
}

\begin{abstract}
A reduced complexity fuzzy model has been developed to capture the nonlinear dynamics of a mechanical system. The use of Functional Principal Analysis to reduce the complexity of the model permitted the use of a linear controller based on that model.
\end{abstract}

Keywords: Fuzzy modelling, Model Predictive Control, Complexity reduction.

\section{Introduction}

Model Predictive Control is considered a well established technology in many fields, especially in industrial process applications. Its efficiency has been proven over many years. In general, most applications of predictive control are based on linear models, which yield good results especially if they work around an operating point [1]. However, there are many applications where the region of operation of the system reduce the prediction capabilities of linear models, leading to poor controller performance. In such cases, Non Linear Model Predictive Control (NMPC) is a suitable option.

Although the number of applications of NMPC is limited [2], its potential is enormous. The possibility of dealing with nonlinear dynamics is the main advantage over MPC. However, developing precise nonlinear models from first principles may be a difficult task in many complex processes. Another disadvantage is that the optimiser solution in non-linear Predictive Control is a non-convex problem and a large computational effort may be required to obtain the solution. This is especially relevant when dealing with real time tasks.

Therefore, industrial control platforms with low computational power can not run nonlinear predictive control strategies.

The strategy proposed in [3-6] involves calculating as many MPC linear controllers $u_{j}(k)$ as linear models obtained in the neurofuzzy model, such that the controller output at the instant $k$ will be (3):

$$
u(k)=\sum_{j=1}^{L} w_{j}(k) u_{j}(k)
$$

where $L$ is the number of linear models and $w_{j}$ is defined as

$$
w_{j}(k)=\frac{\bar{\mu}_{j}(k)}{\sum_{j=1}^{N} \bar{\mu}_{j}(k)}, \quad \bar{\mu}_{j}(k)=\prod_{i=1}^{n} \mu_{i j}(k)
$$

Where $\mu_{i j}(k)$ is the degree of membership of the input $i$ for the rule $j$ Due to the nonlinear nature of the fuzzy system, there are different solutions given to the FMPC optimisation problem. Branch and Bound [7, 8] or Genetic algorithms [8, 9] are used by several authors, others linearise the TS fuzzy model in the operating point, solving a linear MPC problem [1012]. A simpler scheme is used, designing multi-model in the TS fuzzy model [10, 12]. The ability to build fuzzy logic applications for control problems has been hindered by the well-known problem of combinatorial rules explosion, causing complexity in modeling. The existence of redundant rules may also cause performance degradation of the FIS [13]. In this work, we will apply a simplification technique explained in [14], based on Functional Principal Component Analysis (FPCA) to reduce the number of consequents in a fuzzy model in order to design a simpler MPC. This paper is organised as follows. In section 2, a fuzzy model for a nonlinear mechanical system is presented. Functional Principal Component Analysis is described in section 3, where the application to fuzzy systems, using the mechanical system model is illustrated. In section 4, a Model Predictive Controller is designed based on the simplified fuzzy model and it is applied in comparison with other controllers. Conclusions are given in section 5

\section{Fuzzy model for nonlinear systems}

A nonlinear system may be described by a Takagisugeno [15] fuzzy model with $j$ rules by the following way:

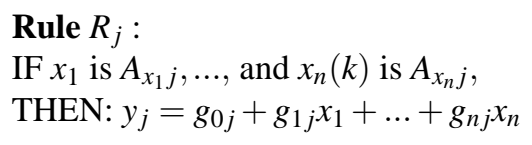

being $x_{i}, y_{j}$ for each rule, the inputs and outputs of the system respectively, and $A_{x_{i}}$ is the fuzzy set respective to $x_{i}(k)$ on the rule $j, g_{i} \in \mathbb{R}, y_{j}(k)$ is the 
output of the model respective to the operating region associated to that rule. The structure of antecedents describes fuzzy regions in the inputs space, and the one of consequents presents non-fuzzy functions of the model inputs.

The output of the model at the time $k$, can be described by

$$
y(k)=\sum_{j=1}^{L} w_{j}(k) y_{j}(k)
$$

Where $w_{j}(k)$ is defined in 2

As an illustrative example we will model the mechanical system shown in figure 1. It could be a simple manipulator with only one joint. The system is moved by an electrical motor which provides a torque $T_{u}$ in order to move a bar of length $l$ an angle $\theta$. If we consider all the mass $(m)$ concentrated at the end of the bar and the friction coefficient $B$, the equation that describes the system is:

$$
m \ddot{\theta} l^{2}+B \dot{\theta}+m g \sin \theta=T_{u}
$$

For simulation the parameters will be: $g=9.8 \mathrm{~m} / \mathrm{s}^{2}$, $l=1 \mathrm{~m}, B=1 \mathrm{Kgm}^{2} / \mathrm{s}, \mathrm{m}=1 \mathrm{Kg}$. As we can observe

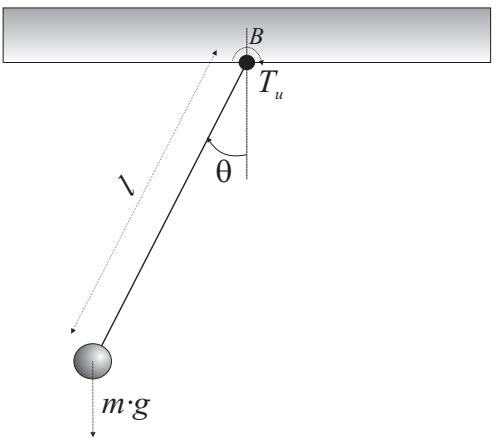

Figure 1: Mechanical system

in (4), the system has a non-linearity due to $\sin \theta$. Linearizing around an equilibrium point, we could model the system as

$$
\ddot{\theta}=-a \dot{\theta}-b \theta+T_{u}
$$

Where $a, b$ are parameters depending on the operating point $\left(\theta_{0}\right)$. It is a second order linear system. In order to build a Fuzzy system, we use four variables in discrete mode, to get the dynamics of a $2^{\text {nd }}$ order system: $T_{u}(k-2), T_{u}(k-1), \theta(k-2), \theta(k-1)$. Taking small steps to the input (torque), we can model the response as second order system (5), different for each operating point determined by the position of the mechanism. Doing this in many areas in order to get enough set of rules to increase the complexity of the FIS, we have several linear systems. In this particular example, nine rules were chosen:

$$
\begin{aligned}
& \theta_{1}(k)= 0.0037 T(k-1)+0.0467 T(k-2) \\
&-0.9705 \theta(k-1)+1.9705 \theta(k-2) \\
& \theta_{2}(k)=-0.0016 T(k-1)+0.0525 T(k-2) \\
&-0.9704 \theta(k-1)+1.9645 \theta(k-2) \\
& \theta_{3}(k)=-0.0001 T(k-1)+0.0508 T(k-2) \\
&-0.9704 \theta(k-1)+1.9628 \theta(k-2) \\
& \theta_{4}(k)=-0.0003 T(k-1)+0.0511 T(k-2) \\
&-0.9704 \theta(k-1)+1.9621 \theta(k-2) \\
& \theta_{5}(k)=-0.0003 T(k-1)+0.0508 T(k-2) \\
&-0.9705 \theta(k-1)+1.9619 \theta(k-2) \\
& \theta_{6}(k)=-0.0003 T(k-1)+0.0510 T(k-2) \\
&-0.9704 \theta(k-1)+1.9621 \theta(k-2) \\
& \theta_{7}(k)=-0.0002 T(k-1)+0.0509 T(k-2) \\
&-0.9704 \theta(k-1)+1.9629 \theta(k-2) \\
& \theta_{8}(k)=-0.0008 T(k-1)+0.0515 T(k-2) \\
&-0.9704 \theta(k-1)+1.9650 \theta(k-2) \\
& \theta_{9}(k)= 0.00065 T(k-1)+0.0501 T(k-2) \\
&-0.9704 \theta(k-1)+1.9705 \theta(k-2)
\end{aligned}
$$

where $\theta_{i}(k)$ is the angle variation for the local model $i$, and $T(k-j)$ is the variation of the applied torque.

Providing data sets from simulations for training and checking, the Fuzzy Inference System (FIS) obtained is defined by the membership function depicted in figure 2. where the universe of discourse are, for the angle $-100^{\circ}<\theta<100^{\circ}$ and for the torque $-10 \mathrm{Nm}<T<$ $10 \mathrm{Nm}$
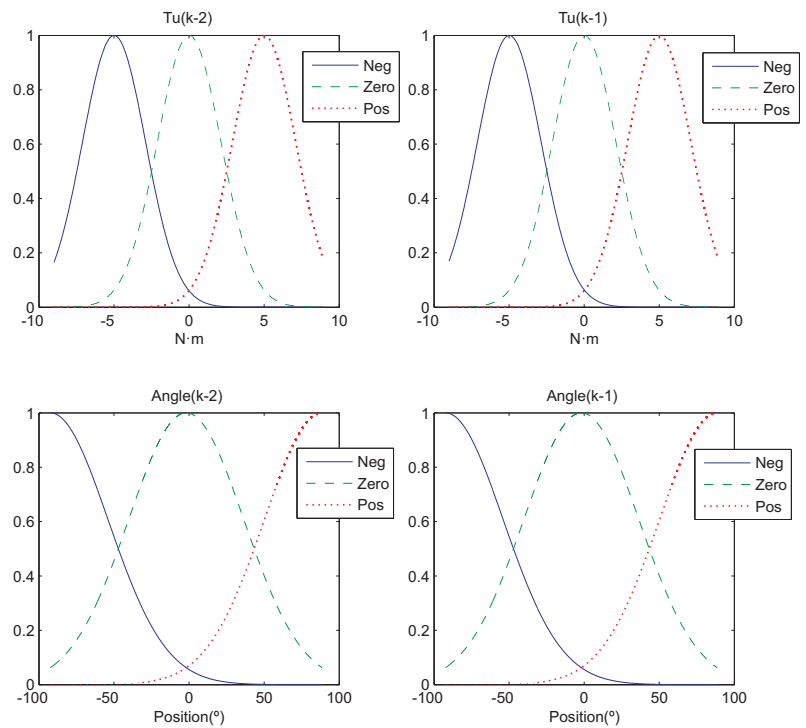

Figure 2: Membership functions for the mechanical system FIS 
establishing rules with variable angle as antecedent, we can model the mechanical system with minimum error, as is shown in fig 3. obtaining a RMSE of $\pm 0.1064^{\circ}$ over 3334 samples, when a white noise is added to the torque action in simulation.

\section{Fuzzy model consequents reduction based on FPCA}

There are well-know methods to reduce complexity of fuzzy systems. Most of them are based on systematic and heuristic methods (e.g. [16, 17], others with analytic approach, are practically unapplicable when the number of inputs is large (eg. [16, 18]. Multivariate Statistics is used in control engineering for many years [19]. Singular Value Decomposition (SVD) techniques such as PCA has been used in control engineering for sensor fault detection [20], variable decoupling [21] and modelling [22, 23]. Dimensionality reduction [24] is the main feature that takes advantage of these techniques.

\subsection{Functional Principal Component Analysis}

Let $f_{1}(x), f_{1}(x), \ldots, f_{n}(x)$ be functions in separable Hilbert space endowed with inner product:

$$
\left\langle f_{i} \mid f_{j}\right\rangle=\int_{0}^{X} f_{i}(x) f_{j}(x) d x, \quad \forall f_{i, j} \in L^{2}[0, X]
$$

If each function $f_{i}(x)$ may be decomposed in:

$$
f_{i}(x)=\sum_{l=1}^{L} c_{i l} \theta_{l}(x)=\mathbf{c}_{\mathbf{i}}^{T} \Theta(x)
$$

The mean and covariance functions of $f_{i}$, will be:

$$
\begin{gathered}
\bar{f}(x)=E(f(x))=\overline{\mathbf{c}}^{T} \Theta(x) \\
\operatorname{Cov}[f(x), f(s)]=\Theta(x)^{T} \operatorname{cov}(\mathbf{C}) \Theta(s)
\end{gathered}
$$

Where $\mathbf{C}=\left\{c_{i l}, i=1, \ldots, n, l=1, \ldots, L\right\}$.

We define the covariance operator as:

$$
\begin{aligned}
C(f(x))= & \int_{0}^{X} \operatorname{Cov}[f(x), f(s)] f(s) d s, \\
& \forall f \in L^{2}[0, X], \forall x, s \in[0, X]
\end{aligned}
$$

Where the kernel $\operatorname{Cov}[f(x), f(s)]$ is the covariance function.

The covariance operator is positive, selfadjoint and compact [25], thus, using Mercer's Theorem, we may write:

$$
\operatorname{Cov}[f(x), f(s)]=\sum_{i=1}^{\infty} \lambda_{i} \xi_{i}(x) \xi_{i}(s), \quad \forall x, s \in[0, X]
$$

where $\lambda_{1}>\lambda_{2}>\ldots>0$ is an enumeration of the eigenvalues of $C$, and the corresponding orthonormal eigenfunctions are $\xi_{1}, \xi_{2}, \ldots$. Thus, they form a complete orthonormal set of solutions of the Fredholm equation:

$$
\int_{0}^{X} \operatorname{Cov}[f(x), f(s)] \xi_{i}(s) d s=\lambda_{i} \xi_{i}(x)
$$

\subsection{FPCA for Fuzzy Inference Systems}

We can formulate the expression 3 as:

$$
y(\mathbf{x})=\tilde{g}_{0}(\mathbf{x})+\tilde{g}_{1}(\mathbf{x}) x_{1}+\ldots+\tilde{g}_{n}(\mathbf{x}) x_{n}
$$

Where:

$$
\tilde{g}_{i}(\mathbf{x})=\sum_{j=1}^{N} a_{j}(\mathbf{x}) \cdot g_{j i}
$$

And the vector of functions $\tilde{\mathbf{g}}$ is:

$$
\begin{gathered}
\tilde{\mathbf{g}}(\mathbf{x})=\left[\begin{array}{c}
\tilde{g}_{0}(\mathbf{x}) \\
\tilde{g}_{1}(\mathbf{x}) \\
\vdots \\
\tilde{g}_{n}(\mathbf{x})
\end{array}\right]=\left[\begin{array}{cccc}
g_{10} & g_{20} & \ldots & g_{N 0} \\
g_{11} & g_{21} & \ldots & g_{N 1} \\
& \vdots & & \\
g_{1 n} & g_{2 n} & \ldots & g_{N n}
\end{array}\right] \cdot\left[\begin{array}{c}
a_{0}(\mathbf{x}) \\
a_{1}(\mathbf{x}) \\
\vdots \\
a_{N}(\mathbf{x})
\end{array}\right] \\
\tilde{\mathbf{g}}(x)=\mathbf{G} \cdot \mathbf{a}(x)
\end{gathered}
$$

The mean and covariance functions of $\tilde{\mathbf{g}}(x)$, are:

$$
\begin{array}{r}
E[\tilde{\mathbf{g}}(x)]=E\left[\mathbf{g}^{T}\right] \cdot \mathbf{a}(x)=\overline{\mathbf{g}}^{T} \cdot \mathbf{a}(x) \\
\operatorname{Cov}[\tilde{\mathbf{g}}(x), \tilde{\mathbf{g}}(s)]=\mathbf{a}(x)^{T} \operatorname{cov}(\mathbf{G}) \mathbf{a}(s)
\end{array}
$$

We have to solve the equation (12), to obtain the FPCA of these functions. We suppose that the eigenfunctions are

$$
\xi(x)=\mathbf{a}(x)^{T} \cdot \mathbf{b}
$$

Thus, taking in account (16):

$$
\begin{array}{r}
\int_{0}^{X} \operatorname{Cov}[\tilde{\mathbf{g}}(x), \tilde{\mathbf{g}}(s)] \cdot \xi(s) d s= \\
\int_{0}^{X} \mathbf{a}(x)^{T} \operatorname{cov}(\mathbf{G}) \mathbf{a}(s) \cdot \mathbf{a}(s)^{T} \cdot \mathbf{b} d s= \\
\mathbf{a}(x)^{T} \operatorname{cov}(\mathbf{G}) \cdot \mathbf{W} \cdot \mathbf{b} \\
\operatorname{cov}(\mathbf{G}) \cdot \mathbf{W} \cdot \mathbf{b}=\lambda \cdot \mathbf{b}
\end{array}
$$

Where:

$$
\mathbf{W}=\int_{0}^{X} \mathbf{a}(s) \cdot \mathbf{a}(s)^{T} d s
$$

The functions $\xi(x)$ are orthogonals, then $\left\langle\xi_{i}(x), \xi_{j}(x)\right\rangle=b_{i}^{T} \cdot \mathbf{W} \cdot b_{j}=0 . \quad$ Matrix $\mathbf{W}$ is symmetric by definition, thus, defining $\mathbf{u}=\mathbf{W}^{\frac{1}{2}} \mathbf{b}$,

$$
\mathbf{W}^{\frac{1}{2}} \cdot \operatorname{cov}(\mathbf{G}) \cdot \mathbf{W}^{\frac{1}{2}} \cdot \mathbf{u}=\lambda \cdot \mathbf{u}
$$

We are left with solving a symmetric eigenvalue problem. Afterward, using a variability criteria, we can choose a new subspace using a new base of eigenfunction whose eigenvalues have enough significance, for instance

$$
\frac{\sum_{i=1}^{l} \lambda_{i}}{\sum_{i=1}^{n} \lambda_{i}} \geq v
$$



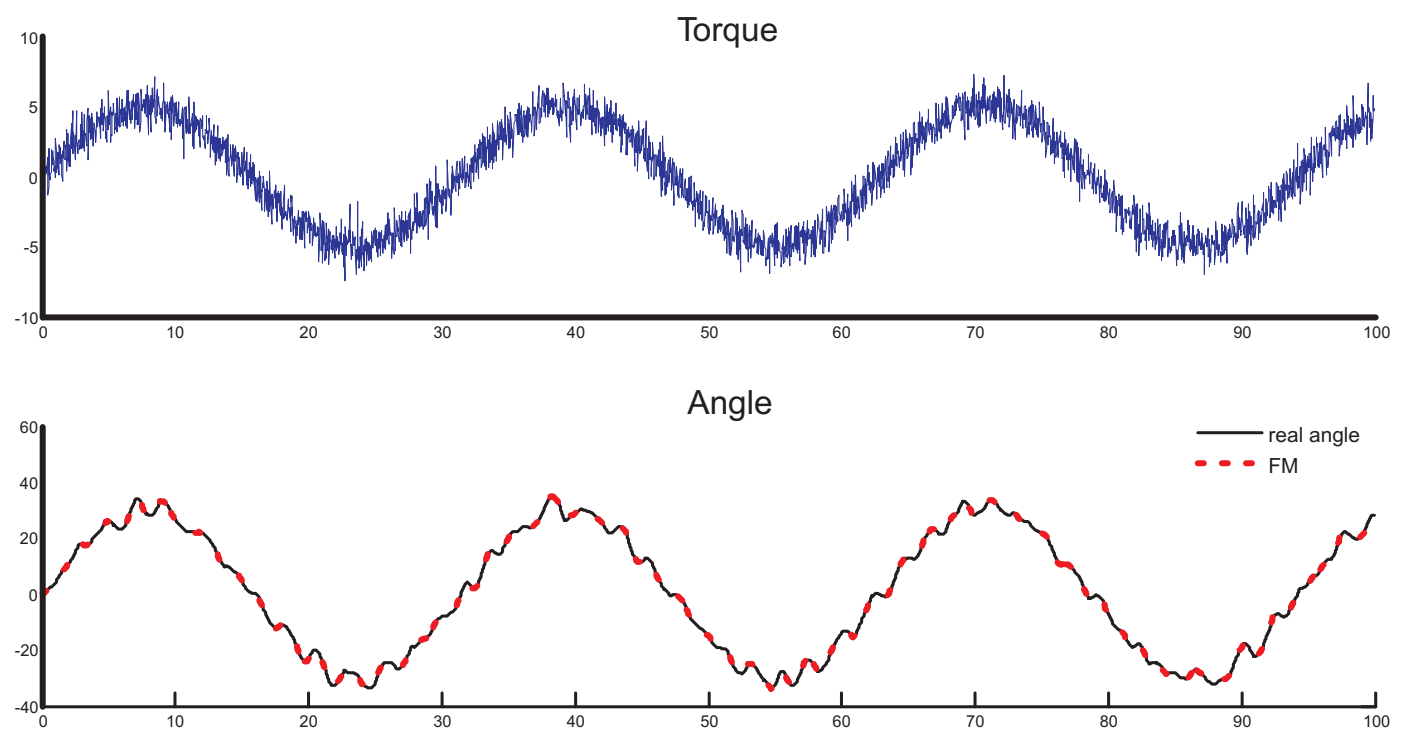

Figure 3: Validation of the fuzzy model for the mechanical system

where $v \in[0,1]$ is the variability index $(v=1$ corresponding to the maximum variability obtained in the new space, i.e. the new subspace has the same dimension of the original space). $N$ is the dimension of the original space and $R$ is for the new reduced subspace.

\subsection{Example: Mechanical system}

Applying the previous reduction technique to the mechanical system modeled by 4 , it is observed that the first eigenvalue contents almost all the variability of the system. Thus, the new simplified system will have just one rule and its structure is given by:

$$
\tilde{\mathbf{g}}(x)=\left(\begin{array}{c}
0 \\
0.0198 \\
-0.3788 \\
0.7669 \\
-0.0034
\end{array}\right) \cdot \xi(x)
$$

And

$$
\xi(x)=\mathbf{a}(x)^{T} \cdot\left(\begin{array}{c}
0.0425 \\
0.0433 \\
0.0433 \\
0.0433 \\
0.0433 \\
0.0433 \\
0.0434 \\
0.0435 \\
0.0444
\end{array}\right)
$$

In the figure 4 we can distinguish differences between the fuzzy and simplified fuzzy models. However, with an RMSE of $\pm 2.1692^{\circ}$ over 3334 samples, it is reasonable to use the simplified model for control or simulation.

\section{Application of FPCA to FMPC without constraints}

Following the same procedure presented in [3-6], for each of the consequents of the fuzzy system used for the model, a linear Generalised Predictive Controller (GPC)[1] can be designed. The advantage of this techniques is the simplistic natural way of translating the GPC to linear spaces (consequent of each rule). In this particular example, only 9 controllers must be designed. However, the problem arises when the number of rules increases. The complexity reduction technique based on FPCA can overcome this problem in an efficient manner. Expression (24) shows the principal component containing the maximum variability and 23), the combination of the new consequent and the principal component. Based on the new consequent, just one GPC design is required. A comparison between three control strategies will be carry out over the mechanical system. The first is a clasical PID, adjusted to work around an operation point, the second is a linear GPC designed over the same point, based on a linear model and the third is a Fuzzy GPC with the reduction of complexity produced by FPCA in the fuzzy model. Figure 5 shows a regular performance, independent of the operating point is observed for the FGPC. This scheme can be seen as a linear controller $u_{L}(k)$ (consequent) modulated by a nonlinear factor $\psi(k)$ (antecedent).

$$
u(k)=\psi(k) u_{L}(k)
$$

The controller $u_{L}(k)$ is designed using (13), and applying FPCA,

$$
\tilde{\mathbf{g}}(x)=\mathbf{G} \cdot \mathbf{a}(x)=\mathbf{H} \cdot \xi(x)
$$



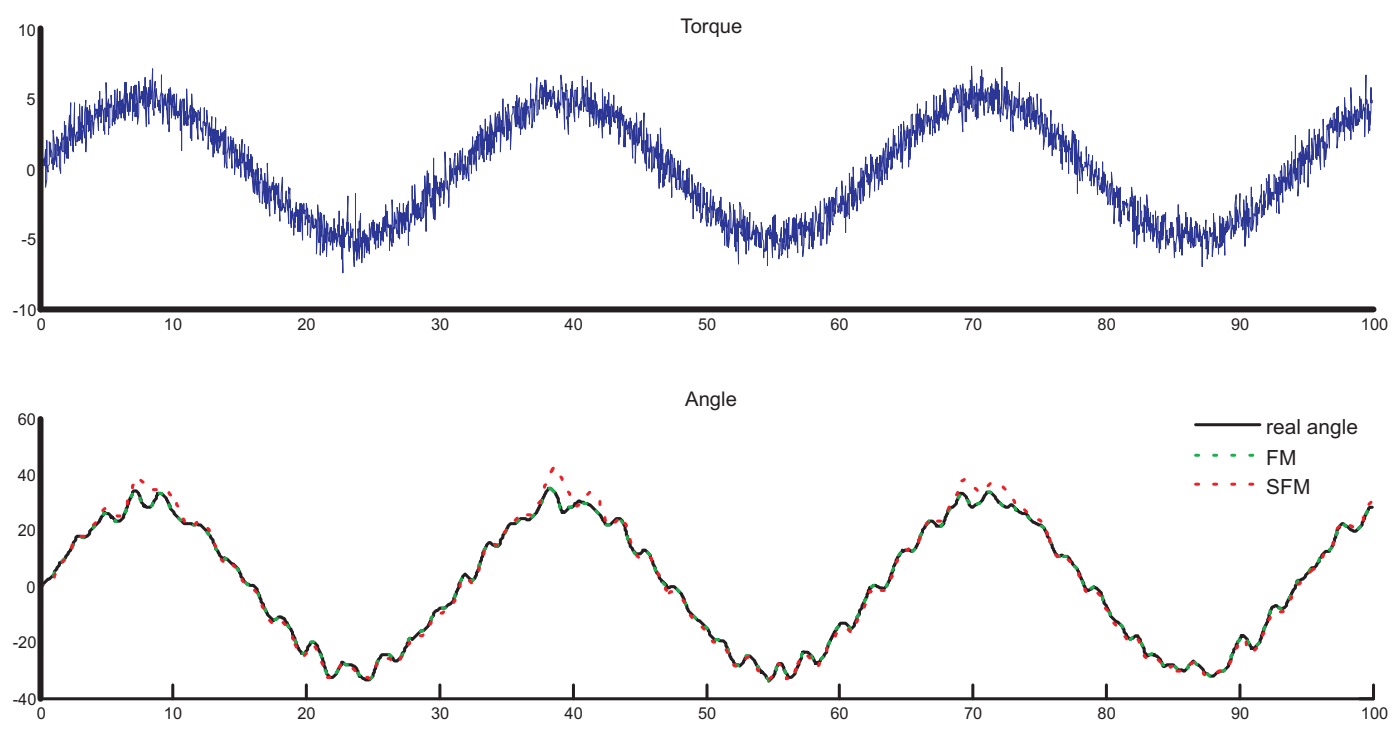

Figure 4: Mechanical system: Comparison between original (real), Fuzzy model (FM) and simplified Fuzzy model (SFM)

Where $\mathbf{H}$ is the new consequent and $\xi(x)$ the antecedent. Knowing (17),

$$
\begin{array}{r}
\mathbf{G} \cdot \mathbf{a}(x)=\mathbf{H} \cdot \mathbf{a}(x) \cdot \mathbf{b}^{T} \\
\mathbf{G} \cdot \mathbf{a}(x) \cdot \mathbf{b}^{T}=\mathbf{H} \cdot \mathbf{a}(x) \cdot \mathbf{b}^{T} \cdot \mathbf{b}^{T} \\
\mathbf{G}=\mathbf{H} \cdot \mathbf{b}^{T} \\
\mathbf{H}=\frac{\mathbf{G} \cdot \mathbf{b}}{\mathbf{b}^{T} \mathbf{b}}
\end{array}
$$

$\frac{1}{\mathbf{b}^{T} \mathbf{b}}$ can be written as $\frac{1}{\mathbf{b}^{T} \mathbf{b}}=\varepsilon \cdot \eta$, having:

$$
\tilde{\mathbf{g}}(x)=\mathbf{H} \cdot \xi(x)=\eta \cdot \mathbf{G} \cdot \mathbf{b} \cdot \varepsilon \cdot \xi(x)
$$

using $\eta \cdot \mathbf{G} \cdot \mathbf{b}$ as a linear model to design a GPC and modulating the nonlinear term $\varepsilon \cdot \xi(x)$, a stable solution can be found. Figure 5 shows the performance of the controllers. One can see clearly how the Simplified Fuzzy MPC can perform better in different duty points.

\section{Conclusion}

A MPC controller has been designed for the position control of a mechanism. A fuzzy model has been developed in order to get the nonlinear dynamics of the system. The model was validated, using a noisy torque input for both, the physic equation and the FIS. A Functional Principal Analysis has been applied to the FIS, reducing its complexity just to one rule, designing one MPC to control the nonlinear system. The performance of this controller has been tested in simulation.

\section{Acknowledgment}

The authors gratefully acknowledge the Spanish Ministry of Economy and Competitivenes for its financial support of part of this work through the grant DPI2013-46912-C2-1.

\section{References}

[1] E. Camacho and C. Bordons, Model Predictive Control, ser. Advanced Textbooks in Control and Signal Processing. Springer, 2007.

[2] T. A. Badgwell, "Review of nonlinear model predictive control applications," in Non-linear Predictive Control: theory and practice, ser. Control, Robotics \& Sensors. Institution of Engineering and Technology, 2001, pp. 3-32.

[3] J. Zhang and A. Morris, "Long range predictive control of nonlinear processes based on recurrent neuro-fuzzy network models," Neural Computing \& Applications, vol. 9, no. 1, pp. 50-59, 2000.

[4] J. J. Espinosa, M. Hadjili, V. Wertz, and J. Vandewalle, "Predictive control using fuzzy models - comparative study," in Proc. European Control Conference, 1999.

[5] S. Townsend and G. W. Irwin, "Nonlinear model based predictive control using multiple local models," IEE Control Engineering series, pp. 223-244, 2001.

[6] J. Soto, I. Castillo, and A. Gauthier, "Identificación y control de sistemas no lineales mediante clustering y gpc usando modelos difusos takagisugeno," Revista de Ingeniería, no. 19, pp. 150157, 2004.

[7] B. Potocnik, G. Music, and B. Zupancic, "Model predictive control systems with discrete inputs," in Electrotechnical Conference, 2004. MELECON 2004. Proceedings of the 12th IEEE Mediterranean, vol. 1, May 2004, pp. 383-386 Vol.1. 

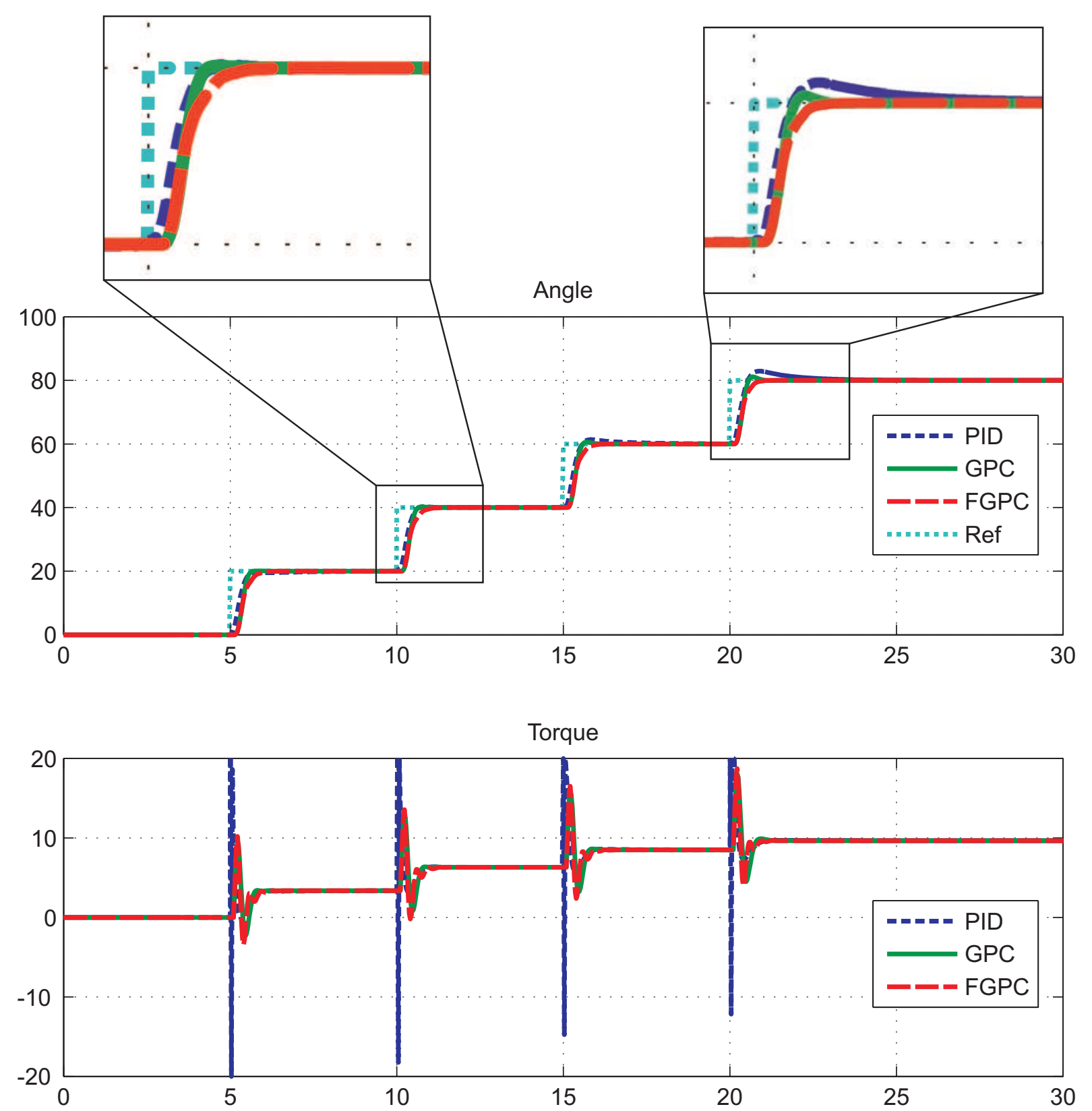

Figure 5: Mechanical system position control comparison 
[8] G. Karer, G. Mušič, I. Škrjanc, and B. Zupančič, "Hybrid fuzzy model-based predictive control of temperature in a batch reactor," Computers \& Chemical Engineering, vol. 31, no. 12, pp. 1552 - 1564, 2007.

[9] A. Núñez, D. Sáez, S. Oblak, and I. Škrjanc, "Fuzzy-model-based hybrid predictive control," \{ISA\} Transactions, vol. 48, no. 1, pp. $24-31$, 2009.

[10] R. Babuska, J. Sousa, and H. Verbruggen, "Predictive control of nonlinear systems based on fuzzy and neural models," European control conference, p. 667, 1999.

[11] P. Marusak and P. Tatjewski, "Stability analysis of nonlinear control systems with unconstrained fuzzy predictive controllers," Archives of Control Sciences, vol. Vol. 12, no. 3, pp. 267-288, 2002.

[12] P. Tatjewski, Advanced control of industrial processes: structures and algorithms, ser. Advances in industrial control. Springer, 2007.

[13] O. Cordón, F. Herrera, F. Hoffmann, L. Magdalena, O. Cordon, F. Herrera, and F. Hoffmann, Genetic fuzzy systems. World Scientific Publishing Company Singapore, 2001.

[14] J. Escaño and C. Bordons, "Neurofuzzy model of an industrial processs, reducing complexity by using principal component analysis," in $X V I$ Congreso Español sobre Tecnologías y Lógica Fuzzy (ESTYLF 2012)., 2012.

[15] T. Takagi and M. Sugeno, "Fuzzy Identification of Systems and Its Applications to Modeling and Control," IEEE Transactions on Systems, Man, and Cybernetics, vol. 15, no. 1, pp. 116-132, Feb. 1985.

[16] A. Gegov, Complexity Management in Fuzzy Systems - A Rule Base Compression Approach, ser. Studies in Fuzziness and Soft Computing. Springer, 2007, vol. 211.

[17] Y.-J. Chen and C.-C. Teng, "Rule combination in a fuzzy neural network," Fuzzy Sets Syst., vol. 82, pp. 161-166, September 1996.

[18] T. J. Ross, Fuzzy Logic with Engineering Applications. John Wiley \& Sons, Aug. 2004.

[19] M. Piovoso and K. Hoo, "The use of multivariate statistics in process control," in The Control Handbook, Second Edition. Informa UK Limited, dec 2010, pp. 16-1-16-20.

[20] R. Dunia and S. J. Qin, "Joint diagnosis of process and sensor faults using principal component analysis," Control Engineering Practice, vol. 6, no. 4, pp. 457 - 469, 1998.

[21] P. B. Deshpande, Multivariable process control. Research Triangle Park, N.C. : Instrument Society of America, 1989, includes bibliographical references.
[22] L. Palma and F. Vieira Coito, "Tuning pca controllers based on manual control data," in Emerging Technologies Factory Automation (ETFA), 2011 IEEE 16th Conference on, Sept 2011, pp. $1-4$.

[23] L. Brito Palma, F. Vieira Coito, P. Sousa Gil, and R. Neves-Silva, "Process control based on pca models," in Emerging Technologies and Factory Automation (ETFA), 2010 IEEE Conference on, Sept 2010, pp. 1-4.

[24] J. Yao, X. Liu, and X. Zhu, "Reduced dimension control based on online recursive principal component analysis," in American Control Conference, 2009. ACC '09., June 2009, pp. 57135718.

[25] J. Deville, "Méthodes statistiques et numériques de l'analyse harmonique," Annales de l'inséé, no. 15, pp. 3, 5-101, Jan.-Apr. 1974. 